\title{
EDITORIAL
}

\section{Soluble guanylate cyclase stimulators as a potential therapy for PAH: enthusiasm, pragmatism and concern}

\author{
E.D. Michelakis
}

D espite recent progress, pulmonary arterial hypertension (PAH) remains without a cure. The impact of the approved drugs on PAH is small and, with the exception of some parenteral therapies, they have little or uncertain effects on haemodynamics, right ventricular (RV) function and survival.

There are two main reasons for this. First, the complexity of this vascular remodelling disease is high and its pathology includes fundamental mechanisms from several other diseases and syndromes, including neoplasia (the anti-apoptotic and pro-proliferative remodelling within the vessel wall), degenerative diseases (the early loss of endothelial cells and microvessels) and inflammation [1]. In addition, a reversal of vascular remodelling will mean little unless it is accompanied by a reversal of $\mathrm{RV}$ failure, which at this point remains clinically unpredictable. Secondly, whatever therapy is developed, it needs to be directed against the unique features of PAH and should be selective for the pulmonary circulation, sparing the often normal systemic vessels [1]. This second factor is perhaps the most challenging. One of the common features of all existing therapies for PAH is that they were originally developed for systemic vascular diseases and it was only after they either failed or determined to be non-profitable, that they were tested on PAH.

Surprisingly, there are several quite promising therapies for PAH which remain untested in humans. One of the main reasons is the lack of continuity and the poor efficiency in translational research [1]. Typically, one team might perform cellular work, another small animal work, another large animal work and another phase 1 trials, before phase 2 and 3 trials are planned. This usually takes many years.

In the current issue of the European Respiratory Journal (ERJ) [2] and a very recent issue of the $E R J$ [3], the same team from Giessen (Germany), in collaboration with scientists from Bayer, presented biochemistry, cellular, small animal and human tissue work, as well as first clinical application in PAH patients, on a new drug, representative of an exciting new class of drugs: nitric oxide (NO)-independent soluble guanylate cyclase (sGC) stimulators and activators [4]. This molecule-to-human approach has been followed at other times in the past by the

STATEMENT OF INTEREST: A statement of interest for E.D. Michelakis can be found at www.erj.ersjournals.com/misc/statements.dtl

CORRESPONDENCE: E.D. Michelakis, University of Alberta, Edmonton, AB, Canada. E-mail: evangelos. michelakis@capitalhealth.ca
Giessen team and explains not only the speed and efficiency but also the comprehensive way in which data on a new drug are presented to the scientific community. To my mind, this should be the model by which new therapies for diseases as challenging as PAH need to be developed and presented.

\section{THE UNIQUENESS OF PULMONARY CIRCULATION}

Low pressure pulmonary circulation is unique in many ways, compared with systemic circulation. While all systemic arteries dilate in response to hypoxia (in order to provide the hypoxic/ ischaemic organ with more blood/oxygen), the pulmonary arteries (PAs) contract to shunt blood away from the hypoxic areas of the lung and maintain systemic ventilation/perfusion match (fig. 1). The mechanism for this hypoxic pulmonary vasoconstriction (HPV) is redox-based [6-9]. One of the bestdeveloped theories proposes that mitochondria are the critical oxygen sensors. In response to hypoxia, they alter the production of reactive oxygen species (ROS); it is the regulation of downstream redox-sensitive effectors (like the plasma membrane potassium channels) that causes the PA constriction. Although there is debate concerning whether ROS increase or decrease in response to hypoxia $[9,10]$, most agree that this mechanism is mitochondria-based $[6,7,9]$. One of the attractive features of this theory is that it might explain why contraction to hypoxia is restricted to the PAs. This is because the mitochondria between the PA and systemic vascular smooth muscle cells (VSMC) are functionally different (fig. 1) $[5,8]$. It is not known whether the mitochondria in the two circulations are different genetically or whether they become different because of their environments. Indeed, the redox status at normoxia between the pulmonary and the systemic circulations is very different. While the resistance PAs are exposed to oxygen $\left(\mathrm{O}_{2}\right)$ levels of $\sim 100$ Torr, the resistance arteries supplying internal organs are exposed to $\mathrm{O}_{2}$ levels of $<50$ Torr [8]. As a result, the baseline production of ROS in the resistance PAs is much higher than the baseline production of ROS in the systemic arteries, i.e. the PAs are oxidised compared with the systemic arteries (fig. 1) [5]. In summary, the redox status of the pulmonary and systemic circulations is quite different in both normoxia and hypoxia. Therefore, therapies that can target this redox difference might achieve selectivity for the pulmonary circulation. None of the existing therapies are redox-based, whereas the new sGC stimulators and activators are!

\section{SGC IS REDOX-SENSITIVE}

While the transmembrane particulate guanylate cyclase is the receptor for the natriuretic peptides, the cytoplasmic sGC is the 

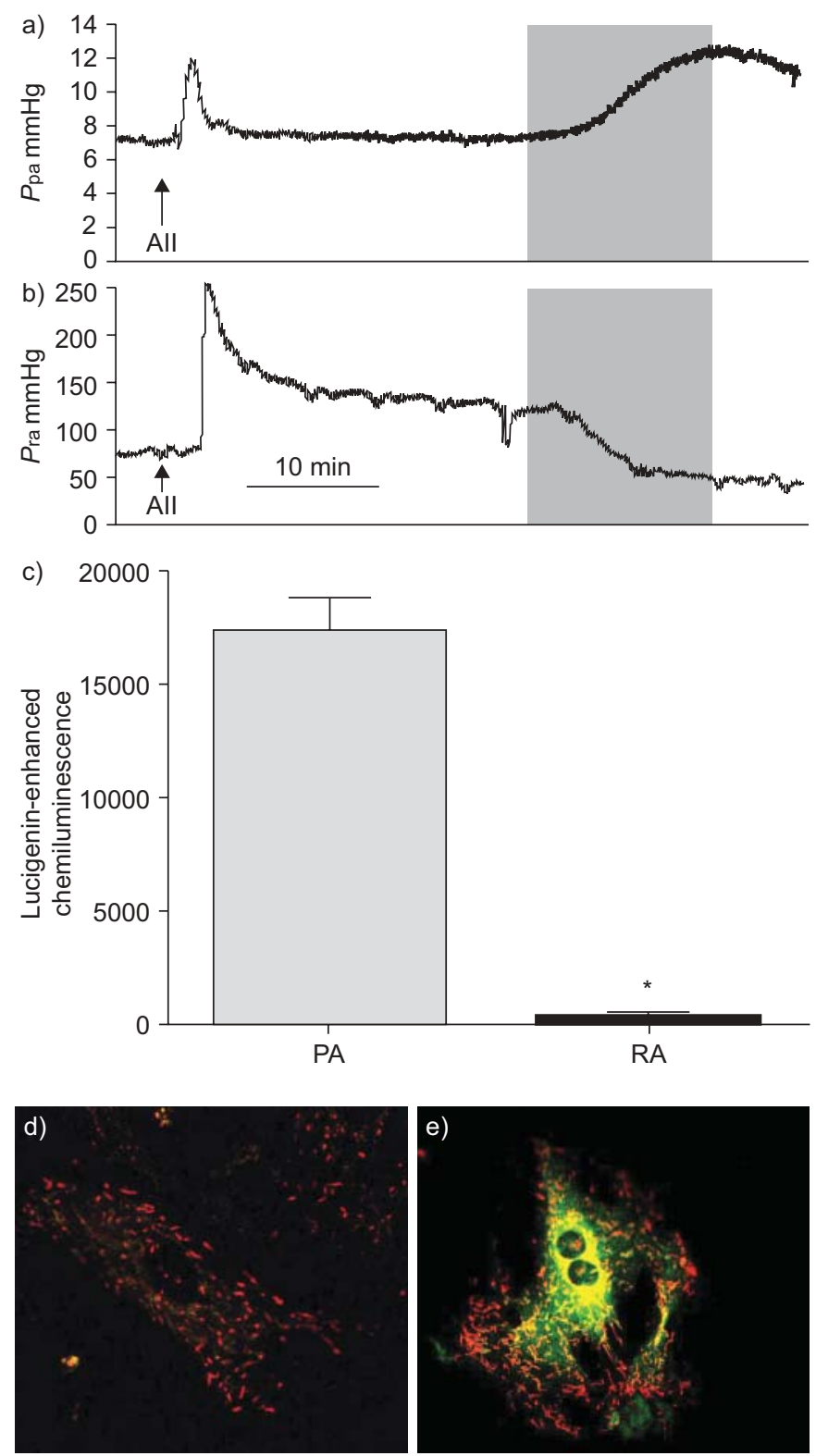

FIGURE 1. In a) rat lungs and b) kidneys perfused in series, induction of hypoxia $(\square)$ causes an increase in pulmonary artery pressure $(P$ pa) and a decrease in renal artery pressure (Pra), while angiotensin II (All) constricts both vascular beds. This is related to the different redox regulation of the two circulations (see main text). c) Even during normoxia, the pulmonary arteries (PAs) are more oxidised than the renal arteries (RAs), as shown by the higher production of reactive oxygen species (ROS) measured by chemiluminescence (photon counts $\cdot \mathrm{s}^{-1}$ ). $d$ and e) The different ROS production at normoxia and the opposing responses to acute hypoxia might be due to the fact that the mitochondria in the arterial smooth muscle cells of the two circulations are different. This is shown by the very different mitochondrial membrane potential (measured using the voltage-sensitive dye JC1) between the d) PA smooth muscle cells and the e) RA smooth muscle cells, even though they are studied under identical conditions. ${ }^{*}: p<0.05$. Reproduced and modified from [5] with permission from the publisher.

receptor for the gaseous signalling molecules carbon monoxide (CO) and NO. In response to endothelial-derived or exogenous $\mathrm{NO}$, the sGC is activated, leading to the production of cyclic guanosine monophosphate (cGMP), which in turn activates protein kinase $G$ with many downstream targets. In the vasculature this typically results in vasodilation. The presence of a reduced $(\mathrm{Fe}++)$ haem moiety is a prerequisite for the NOinduced activation of the sGC. Upon removal of the haem moiety or upon its oxidation, sGC becomes unresponsive to NO. In other words, oxidation of the sGC makes it unresponsive to $\mathrm{NO}$ and in that sense, sGC can be seen as a redox sensor (fig. 2) [4].

Recently, classes of drugs have been developed that can activate sGC independently of NO [4]. The sGC stimulators (e.g. BAY-420-2272) can stimulate the sGC directly and enhance the sensitivity of the reduced enzyme to low levels of NO; thus, sGC stimulators do not affect the oxidised sGC. Conversely, sGC activators (e.g. BAY-58-2667) can directly activate only the haem-free or the oxidised sGC (fig. 2). These drugs: are small molecules; are orally available; are powerful vasodilators; and are not subject to tolerance that complicates the use of exogenous nitrates [4]. The fact that these drugs can selectively target oxidised or reduced vascular environments offers the possibility for selectivity to the diseased tissues. For example, an sGC activator can dilate vessels in diabetic models (which are oxidised due to the disease-generated oxidative stress), sparing normal vessels [11]. Can this be applied to the diseased pulmonary circulation and enhance selectivity?

As previously discussed, it is unclear whether hypoxia causes an increase or a decrease in ROS. According to our group and others, hypoxia (acute and chronic) causes a decrease in ROS, i.e. it reduces the pulmonary circulation $[6,12,13]$. Other studies demonstrate that hypoxia increases ROS, oxidising the PAs [7, 9]. Although these findings may be relevant to hypoxia-induced chronic pulmonary hypertension, they might not be relevant to PAH, a syndrome that is not typically associated with hypoxia. In animal and human PAH PAs, my colleagues and I have shown that the VSMC mitochondria are hyperpolarised (similar to hypoxic VSMC and cancer mitochondria) [12, 14-17]. We have proposed that this hyperpolarisation offers a resistance to apoptosis since the mitochondrial transition pore (from which pro-apoptotic mediators efflux in apoptosis) is mitochondrial membrane potential-sensitive and its opening is associated with depolarisation [1]. We have also shown that this mitochondria hyperpolarisation is associated with a decrease in the production of mitochondrial ROS and that mitochondria-targeting therapies reverse this mitochondrial remodelling and reverse PAH in multiple models [12, 1418]. In other words, PAH PAs are also in a reduced environment, though they could still be relatively more oxidised than the systemic vessels. Although other ROSproducing systems can regulate the cell's redox status, recent evidence suggests that mitochondria are more important than oxidases in regulating redox status in hypoxic PA VSMC and that the opposite is true in hypoxic systemic arteries [19]. These findings suggest that in normoxic baseline, the pulmonary circulation will be less responsive to sGC stimulators than the systemic circulation. However, if we are correct (hypoxia=decreased ROS), the diseased PAs will become responsive to sGC stimulators, like the systemic arteries. If we are wrong (hypoxia=increased ROS), then the diseased PAs will become less responsive to sGC stimulators compared with systemic arteries (fig. 2). 
a)
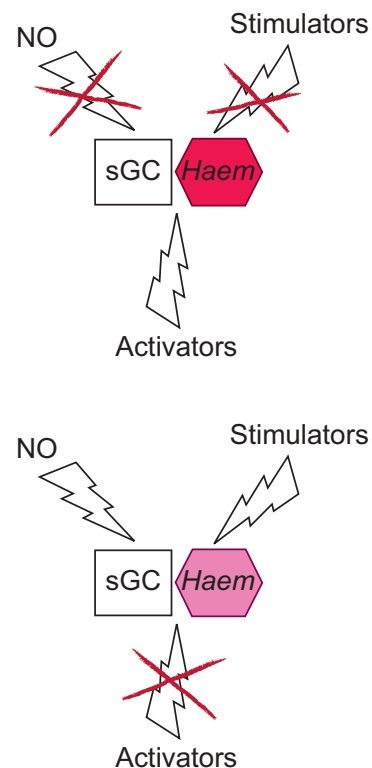

Normoxia
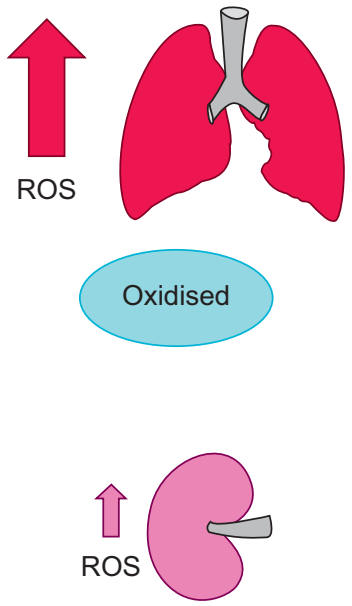

Reduced b)

Hypoxia, PAH
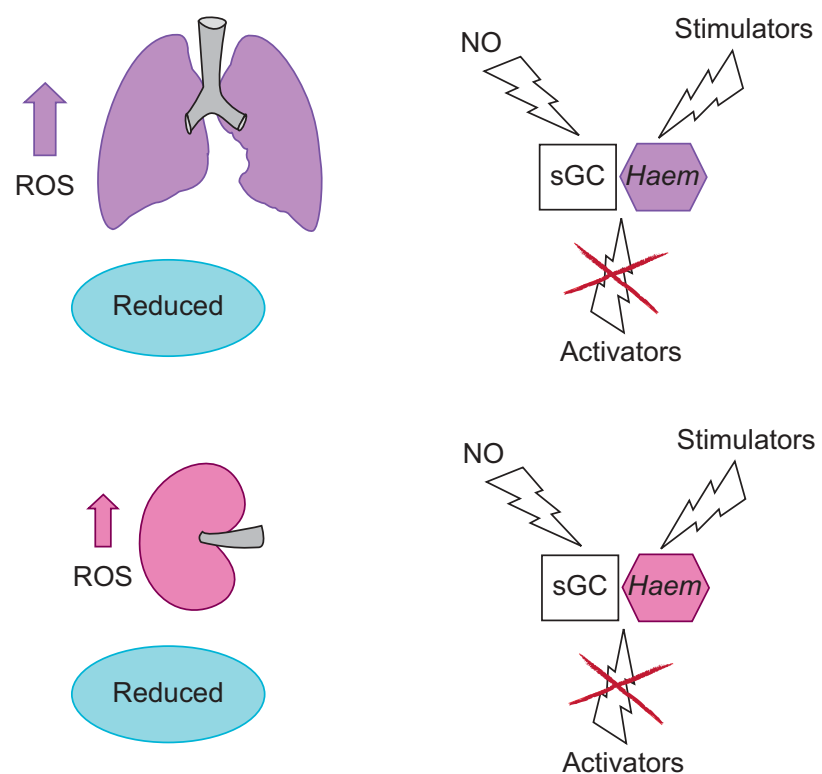

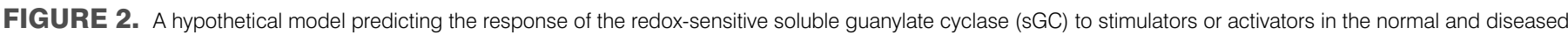
pulmonary versus systemic (i.e. renal) circulation (see text). NO: nitric oxide; ROS: reactive oxygen species; PAH: pulmonary arterial hypertension.

\section{SGC TARGETING IN PAH}

The Giessen group first demonstrated that sGC is highly expressed in the vascular wall of the PAs from PAH patients, although it appears that it is not upregulated compared with normal human lungs [3]. They showed that riociguat, an sGC stimulator (BAY-63-2521), directly activated purified sGC. While an NO donor activated sGC by 25 -fold, the addition of riociguat resulted in a 112-fold activation [3]. The drug reduced acute HPV in isolated mouse lungs; it also decreased acute HPV in lungs from chronically hypoxic mice, more so than in lungs from normoxic mice (perhaps because the chronically hypoxic lungs were more reduced). Riociguat also reversed established monocrotaline rat $\mathrm{PAH}$ and this was associated with a decrease in PA vascular remodelling. The effects of riociguat were immediate, as shown by telemetry catheters, indicating that there was active constriction in that model [3]. Thus, the improvement in the PA remodelling might not be a primary effect but may simply be due to the decrease in the PA pressure. The authors reported that the treatment did not significantly decrease systemic arterial pressure in animals, although there are several limitations in the overall methodology of measuring arterial pressure in rodents. These encouraging animal results were translated to a phase II trial in PAH by the same group, published in the present issue of the ERJ [2].

GRIMMINGER et al. [2] compared the acute effects of 10-20 ppm inhaled nitric oxide (iNO) to increasing oral doses of riociguat on pulmonary and systemic haemodynamics. Although the doses of riociguat cannot be directly compared with the dose of iNO used (up to $20 \mathrm{ppm}$, i.e. not an iNO dose response, since up to $80 \mathrm{ppm}$ iNO are used in $\mathrm{PAH}$ without significant systemic haemodynamic effects), the same group showed that in vitro, the activity of riociguat in activating purified sGC is many-fold higher compared with NO [2]. GRIMMINGER et al. [2] showed that the 5-mg dose had significant haemodynamic effects, but in one of the two patients tested, there was significant hypotension; thus, they focused on the further study of the 1- and 2.5-mg doses. The effects of both doses in pulmonary haemodynamics were modest since the decrease in mean PA pressure was only $\sim 5.1 \mathrm{mmHg}$ for the $2.5-\mathrm{mg}$ dose. Importantly, the decrease in systolic blood pressure was higher, $\sim 29 \mathrm{mmHg}$. Thus, the associated increase in the cardiac index (CI) was a result of vasodilation, mainly on systemic vascular beds.

The fact that the systemic haemodynamic effects of the drug were significant is supported by the increase in heart rate (HR), by $\sim 21 \%$ in the $2.5-\mathrm{mg}$ dose. In fact, in a phase I study of riociguat in healthy volunteers [20], the systemic blood pressure was decreased and the HR was increased in a dose-dependent manner, although this increase was lower in magnitude than in the current phase II study [2]. The fact that the systemic blood pressure did not decrease further and did not become symptomatic in the study by GRIMMINGER et al. [2] was due in part to the secondary significant increase in HR and CI. In many patients with severe PAH and RV failure, this increase might not be possible. Often, these patients have maximal CI with baseline tachycardia and the cardiac reserve is minimal.

In summary, sGC stimulators might be more potent than iNO and may be more useful than phosphodiesterase (PDE)5 inhibitors in conditions in which the baseline production of $\mathrm{NO}$ is decreased, such as in the PAH and its associated PA endothelial dysfunction. However, although redox-sensitive, they appear to suffer from luck of selectivity to the pulmonary circulation, a critical feature of all promising candidate $\mathrm{PAH}$ therapies. This could be because the diseased pulmonary 
circulation is similar to the systemic circulation in terms of oxidative stress (fig. 2).

In addition, no matter how much the cGMP is increased by an activated sGC, it can be degraded downstream by PDEs [21], and we know PDE5 is increased in both the PA [22] and the RV [23] in PAH. Therefore, a combination with PDE5 inhibitor is logical and might theoretically achieve more selectivity for the pulmonary circulation. However, we shouldn't forget the early sildenafil-related deaths, which were due to the severe hypotension occurring when taken along with nitrates. Given the significant systemic effects, the possibility of an even further decrease in systemic pressures is not to be ignored if sGC stimulators are used in combination with PDE5 inhibitors. Inhaled sGC stimulators in combination with oral PDE5 inhibitors might be an attractive possibility [24].

Given the fact that the systemic effects of these drugs were obvious in the phase I study [3] and confirmed in the phase II study [2], it is difficult to understand why Bayer is planning their development in PAH (including idiopathic PAH) and thromboembolic PAH [25]. The powerful vasodilating effects of sGC stimulators or activators and the ability to offer selectivity in diseased vessels (e.g. in diabetic vessels) and their promising effects in animal congestive heart failure (CHF) $[26,27]$, would suggest that they might be better used in systemic vascular disorders, many of which are in fact associated with secondary PH. In fact, their "balanced" ability to decrease both pulmonary vascular resistance and systemic vascular resistance could be a strength rather than a weakness, as in PAH. Due to the neurohormonal activation in $\mathrm{CHF}$, the systemic vascular resistance is elevated and is the target of most current $\mathrm{CHF}$ therapies. There are subgroups of CHF in which the pulmonary vascular resistance is elevated and often "out of proportion" to the left sided filling pressures. In such patients, this "secondary" pulmonary hypertension is a major cause of morbidity. One such group is patients with pulmonary hypertension due to mitral regurgitation, where in some cases PHT remains severe even after the correction of the valve. Another group is patients with $\mathrm{CHF}$ and pulmonary hypertension due to diastolic left ventricular dysfunction or left ventricular hypertrophy. This latter group might actually account for the most common referral in pulmonary hypertension clinics, at least in North America and Europe. Why not test riociguat in these conditions in which a decrease in both pulmonary vascular resistance and systemic vascular resistance might be beneficial?

The PAH field should not be seen as an "easy ride" for the industry. PAH is not an orphan disease any more. The unfortunate early approval of drugs that were not very effective in this disease (but very expensive and not without toxicities), perhaps because the field and the patients were desperate, makes it easier to develop newer drugs. For example, trials with much lower sample sizes than those used for other cardiovascular diseases (in the hundreds), and typically for very short periods of time (3 months), with soft end-points (6-min walk, time to clinical worsening) and without haemodynamic studies, make it "easy". It also appears that it is quite easy to show an improvement of 30$40 \mathrm{~min}$ in the 6-min walk; in the end, even exercise rehabilitation programmes can achieve more than that [28]. If studied in
PAH, nonselective vasodilators need to be assessed carefully using trials that will include invasive haemodynamic and imaging end-points, going beyond single-centre phase II trials.

In summary, GRIMMINGER et al. [2] should be congratulated for their efficient animal-to-human translational work. The scientists at Bayer should be proud that they have discovered amazing and powerful drugs which could have a significant impact on cardiovascular diseases, including, perhaps, the very common secondary pulmonary hypertension. Whether pulmonary arterial hypertension is the best "home" for the development of this class of drug remains questionable.

\section{REFERENCES}

1 Michelakis ED, Wilkins MR, Rabinovitch M. Emerging concepts and translational priorities in pulmonary arterial hypertension. Circulation 2008; 118: 1486-1495.

2 Grimminger F, Weimann G, Frey R, et al. First acute haemodynamic study of soluble guanylate cyclase stimulator riociguat in pulmonary hypertension. Eur Respir J 2009; 33: 785-792.

3 Schermuly RT, Stasch JP, Pullamsetti SS, et al. Expression and function of soluble guanylate cyclase in pulmonary arterial hypertension. Eur Respir J 2008; 32: 881-891.

4 Evgenov OV, Pacher P, Schmidt PM, Hasko G, Schmidt HH, Stasch JP. NO-independent stimulators and activators of soluble guanylate cyclase: discovery and therapeutic potential. Nat Rev Drug Discov 2006; 5: 755-768.

5 Michelakis ED, Hampl V, Nsair A, et al. Diversity in mitochondrial function explains differences in vascular oxygen sensing. Circ Res 2002; 90: 1307-1315.

6 Weir EK, Lopez-Barneo J, Buckler KJ, Archer SL. Acute oxygen-sensing mechanisms. N Engl J Med 2005; 353: 2042-2055.

7 Weissmann N, Sommer N, Schermuly RT, Ghofrani HA, Seeger W, Grimminger F. Oxygen sensors in hypoxic pulmonary vasoconstriction. Cardiovasc Res 2006; 71: 620-629.

8 Michelakis ED, Weir EK. The metabolic basis of vascular oxygen sensing: diversity, compartmentalization, and lessons from cancer. Am J Physiol Heart Circ Physiol 2008; 295: H928-H930.

9 Guzy RD, Schumacker PT. Oxygen sensing by mitochondria at complex III: the paradox of increased reactive oxygen species during hypoxia. Exp Physiol 2006; 91: 807-819.

10 Weir EK., Archer SL. Counterpoint: hypoxic pulmonary vasoconstriction is not mediated by increased production of reactive oxygen species. J Appl Physiol 2006; 101: 995-998; discussion 998.

11 Stasch JP, Schmidt PM, Nedvetsky PI, et al. Targeting the heme-oxidized nitric oxide receptor for selective vasodilatation of diseased blood vessels. J Clin Invest 2006; 116: 2552-2561.

12 Michelakis ED, McMurtry MS, Wu XC, et al. Dichloroacetate, a metabolic modulator, prevents and reverses chronic hypoxic pulmonary hypertension in rats: role of increased expression and activity of voltage-gated potassium channels. Circulation 2002; 105: 244-250.

13 Michelakis ED, Thebaud B, Weir EK, Archer SL. Hypoxic pulmonary vasoconstriction: redox regulation of $\mathrm{O}_{2}$-sensitive 
$\mathrm{K}+$ channels by a mitochondrial $\mathrm{O}_{2}$-sensor in resistance artery smooth muscle cells. J Mol Cell Cardiol 2004; 37: 1119-1136.

14 Bonnet S, Archer SL, Allalunis-Turner J, et al. A mitochondria- $\mathrm{K}+$ channel axis is suppressed in cancer and its normalization promotes apoptosis and inhibits cancer growth. Cancer Cell 2007; 11: 37-51.

15 Bonnet S, Michelakis ED, Porter CJ, et al. An abnormal mitochondrial-hypoxia inducible factor- $1 \alpha-\mathrm{Kv}$ channel pathway disrupts oxygen sensing and triggers pulmonary arterial hypertension in fawn hooded rats: similarities to human pulmonary arterial hypertension. Circulation 2006; 113: 2630-2641.

16 McMurtry MS, Archer SL, Altieri DC, et al. Gene therapy targeting survivin selectively induces pulmonary vascular apoptosis and reverses pulmonary arterial hypertension. $J$ Clin Invest 2005; 115: 1479-1491.

17 McMurtry MS, Bonnet S, Wu X, et al. Dichloroacetate prevents and reverses pulmonary hypertension by inducing pulmonary artery smooth muscle cell apoptosis. Circ Res 2004; 95: 830-840.

18 Bonnet S, Rochefort G, Sutendra G, et al. The nuclear factor of activated $\mathrm{T}$ cells in pulmonary arterial hypertension can be therapeutically targeted. Proc Natl Acad Sci USA 2007; 104: 11418-11423.

19 Mehta JP, Campian JL, Guardiola J, Cabrera JA, Weir EK, Eaton JW. Generation of oxidants by hypoxic human pulmonary and coronary smooth-muscle cells. Chest 2008; 133: 1410-1414.

20 Frey R, Muck W, Unger S, Artmeier-Brandt U, Weimann G, Wensing G. Pharmacokinetics, pharmacodynamics, tolerability, and safety of the soluble guanylate cyclase activator cinaciguat (BAY 58-2667) in healthy male volunteers. J Clin Pharmacol 2008; 48: 1400-1410.

21 Michelakis ED. The role of the NO axis and its therapeutic implications in pulmonary arterial hypertension. Heart Fail Rev 2003; 8: 5-21.

22 Wharton J, Strange JW, Moller GM, et al. Antiproliferative effects of phosphodiesterase type 5 inhibition in human pulmonary artery cells. Am J Respir Crit Care Med 2005; 172: 105-113.

23 Nagendran J, Archer SL, Soliman D, et al. Phosphodiesterase type 5 is highly expressed in the hypertrophied human right ventricle, and acute inhibition of phosphodiesterase type 5 improves contractility. Circulation 2007; 116: 238-248.

24 Evgenov OV, Kohane DS, Bloch KD, et al. Inhaled agonists of soluble guanylate cyclase induce selective pulmonary vasodilation. Am J Respir Crit Care Med 2007; 176: 1138-1145.

25 Investor News 2008. www.investor.bayer.de/no_cache/en/ news/achieve/investor-news. Date last accessed: January 15, 2008.

26 Boerrigter G, Costello-Boerrigter LC, Cataliotti A, Lapp H, Stasch JP, Burnett JC Jr. Targeting heme-oxidized soluble guanylate cyclase in experimental heart failure Hypertension 2007; 49: 1128-1133.

27 Boerrigter G, Costello-Boerrigter LC, Cataliotti A, et al. Cardiorenal and humoral properties of a novel direct soluble guanylate cyclase stimulator BAY 41-2272 in experimental congestive heart failure. Circulation 2003; 107: 686-689.

28 Mereles D, Ehlken N, Kreuscher S, et al. Exercise and respiratory training improve exercise capacity and quality of life in patients with severe chronic pulmonary hypertension. Circulation 2006; 114: 1482-1489. 